\title{
Negatively invariant sets and entire trajectories of set-valued dynamical systems
}

\author{
Peter E. Kloeden • Pedro Marín-Rubio
}

Received: date / Accepted: date

\begin{abstract}
Strongly negatively invariant compact sets of set-valued autonomous and nonautonomous dynamical systems on a complete metric space, the latter formulated in terms of processes, are shown to contain a weakly positively invariant family and hence entire solutions. For completeness the strongly positively invariant case is also considered, where the obtained invariant family is strongly invariant. Both discrete and continuous time systems are treated. In the nonautonomous case, the various types of invariant families are in fact composed of subsets of the state space that are mapped onto each other by the set-valued process. A simple example shows the usefulness of the result for showing the occurrence of a bifurcation in a set-valued dynamical system.
\end{abstract}

Keywords entire solutions - invariant sets · positively invariant sets · negatively invariant sets · set-valued dynamical systems · autonomous and nonautonomous dynamical systems $\cdot$ set-valued processes.

Mathematics Subject Classification (2000) 37B55 - 37L25 - 54C60 - 37G10 . 39A41.

\section{Introduction}

Let $\phi$ be a single-valued autonomous semi-dynamical system on a complete metric space $\left(X, d_{X}\right)$ and let $A$ be a nonempty compact subset of $X$ which is $\phi$-invariant, i.e. $\phi(t, A)=A$ for all $t \in \mathbb{R}^{+}$. It is known that there exists at least one entire trajectory through each point $a \in A$ that is contained in $A$, i.e. a $\chi: \mathbb{R} \rightarrow X$ such that $\chi(t+s)$ $=\phi(s, \chi(t))$ for all $t \in \mathbb{R}$ and $s \in \mathbb{R}^{+}$, with $\chi(0)=a$ and $\chi(t) \in A$ for all $t \in \mathbb{R}$.

Peter E. Kloeden

Institut für Mathematik, Johann Wolfgang Goethe Universität, D-60054 Frankfurt am Main, Germany

E-mail: kloeden@math.uni-frankfurt.de

Pedro Marín-Rubio

Departamento de Ecuaciones Diferenciales y Análisis Numérico, Universidad de Sevilla, Apdo. de Correos 1160, 41080-Sevilla, Spain

E-mail: pmr@us.es 
Positively invariant sets are often encountered as absorbing sets, which is a first step in order to prove the existence of an attractor. Negatively invariant sets are not discussed directly so often in the literature (e.g. cf. [18]), but are present in many unstable situations such as following the loss of stability in a bifurcation or on an unstable manifold about an equilibrium point, e.g. cf. [3,5,6]. In [13] it was showed that similar results hold for positively invariant and negatively invariant compact subsets. Both autonomous and nonautonomous systems with discrete and continuous time sets were considered. In the nonautonomous case, the various types of invariant objects are in fact families of subsets of the state space that are mapped onto each other by the nonautonomous process.

In this paper we establish analogous results for set-valued dynamical systems, both autonomous and nonautonomous. The (strongly) positively invariant case corresponds to the single-valued framework discussed in [13]. However, in the (strongly) negatively invariant case the invariant object obtained is only weakly positively invariant rather than strongly invariant, but this suffices for the construction of entire trajectories. The main technical difficulty arises in the case of strongly negatively invariant subsets for continuous time systems due to the fact that a trajectory joining two points in the set may leave it at intermediate times. To overcome this a limiting argument is used that involves the systems frozen at discrete dyadic times.

The following notation will be used through the paper: given a metric space $\left(X, d_{X}\right)$, let $\mathcal{K}(X)$ be the family of all nonempty compact subsets of $\left(X, d_{X}\right)$. Then, for sets $A$ and $B \in \mathcal{K}(X)$ dist $_{X}(\cdot, \cdot)$ will denote the Hausdorff semi-distance, i.e.

$$
\operatorname{dist}_{X}(A, B)=\sup _{a \in A} \inf _{b \in B} d_{X}(a, b),
$$

and $H_{X}(\cdot, \cdot)$ will denote the Hausdorff metric, i.e.

$$
H_{X}(A, B)=\max \left\{\operatorname{dist}_{X}(A, B), \operatorname{dist}_{X}(B, A)\right\} .
$$

Indeed $\mathcal{K}(X)$ is itself a complete metric space with the Hausdorff metric $H_{X}$.

To allow for both continuous and discrete time systems, we let $\mathbb{T}$ be either $\mathbb{R}$ or $\mathbb{Z}$, $\mathbb{T}^{+}=\mathbb{T} \cap \mathbb{R}^{+}$, and $\mathbb{T}_{2}^{\geq}:=\left\{(t, s) \in \mathbb{T}^{2}: t \geq s\right\}$.

The following well known result will be used repeatedly.

Lemma 1 Let $\left\{A_{n}, n \in \mathbb{N}\right\}$, be a nested sequence of nonempty compact subsets of a complete metric space $\left(X, d_{X}\right)$. Then $A=\cap_{n \geq 1} A_{n}$ is a nonempty compact subset of $X$ and

$$
\operatorname{dist}_{X}\left(A_{n}, A\right) \rightarrow 0 \quad \text { as } n \rightarrow \infty
$$

\section{Single-valued systems}

We consider systems on a complete metric space $\left(X, d_{X}\right)$ as the state space.

2.1 Autonomous systems

The following definition is well known, see for instance [7,23]. 
Definition 1 An autonomous semi-dynamical system on $\left(X, d_{X}\right)$ is a continuous mapping $\mathbb{T}^{+} \times X \ni\left(t, x_{0}\right) \mapsto \phi\left(t, x_{0}\right) \in X$ with the initial value and semi-group properties

(i) $\phi\left(0, x_{0}\right)=x_{0}$ for all $x_{0} \in X$,

(ii) $\phi\left(s+t, x_{0}\right)=\phi\left(s, \phi\left(t, x_{0}\right)\right)$ for all $s, t \in \mathbb{T}^{+}$and $x_{0} \in X$.

The following result was proved in [13].

Theorem 1 Let $A$ be a nonempty compact subset of $X$ which is $\phi$-positively invariant, i.e. $\phi(t, A) \subset A$ for all $t \in \mathbb{T}^{+}$, or $\phi$-negatively invariant, i.e. $A \subset \varphi(t, A)$ for all $t \in$ $\mathbb{T}^{+}$. Then there exists a nonempty compact subset $A_{\infty}$ of $A$ which is $\varphi$-invariant, i.e. $\varphi\left(t, A_{\infty}\right)=A_{\infty}$ for all $t \in \mathbb{R}^{+}$.

\subsection{Nonautonomous systems}

There are two abstract formulations of nonautonomous dynamical systems on a state space $X$, one in terms of skew product flows and the other in terms of processes. The process formulation will be used here, see [4,7]. For the skew product case see [14].

Definition 2 A process is a continuous mapping $\mathbb{T}_{2}^{\geq} \times X \ni\left(t, t_{0}, x_{0}\right) \mapsto \phi\left(t, t_{0}, x_{0}\right) \in X$ with the initial value and evolution properties

(i) $\phi\left(t_{0}, t_{0}, x_{0}\right)=x_{0}$ for all $t_{0} \in \mathbb{T}$ and $x_{0} \in X$,

(ii) $\phi\left(t_{2}, t_{0}, x_{0}\right)=\phi\left(t_{2}, t_{1}, \phi\left(t_{1}, t_{0}, x_{0}\right)\right)$ for all $t_{0} \leq t_{1} \leq t_{2}$ in $\mathbb{T}$ and $x_{0} \in X$.

For greater generality and applicability, invariance properties for nonautonomous systems are usually given in terms of a family of nonempty compact sets $\mathcal{A}=\{A(t), t \in$ $\mathbb{T}$ \} of $X$. The following result was also proved in [13].

Theorem 2 Let $\mathcal{A}=\{A(t): t \in \mathbb{T}\}$ be a family of nonempty compact subsets of $X$ which is positively invariant for a process $\phi$ on $X$, i.e. $\phi\left(t, t_{0}, A\left(t_{0}\right)\right) \subset A(t)$ for all $\left(t, t_{0}\right) \in \mathbb{T}_{2}^{\geq}$, or negatively invariant for the process $\phi$, i.e. $A(t) \subset \phi\left(t, t_{0}, A\left(t_{0}\right)\right)$ for all $\left(t, t_{0}\right) \in \mathbb{T}_{2}^{\geq}$. Then there exists a family of nonempty compact subsets $\mathcal{A}_{\infty}=$ $\left\{A_{\infty}(t): t \in \mathbb{T}\right\}$ of $A$, which is $\phi$-invariant, i.e. $\phi\left(t, t_{0}, A_{\infty}\left(t_{0}\right)\right)=A_{\infty}(t)$ for all $\left(t, t_{0}\right)$ $\in \mathbb{T}_{2}^{\geq}$

Remark 1 For a $\phi$-invariant family of nonempty compact sets $\mathcal{A}=\{A(t): t \in \mathbb{R}\}$ of a continuous time process, it follows that the continuous time set-valued mapping $t \mapsto$ $A(t)=\phi\left(t, t_{0}, A\left(t_{0}\right)\right)$ is continuous in $t \in \mathbb{R}$ with respect to the Hausdorff metric $H_{X}$.

\section{Set-valued dynamical systems}

The above results also hold for set-valued dynamical systems with some modifications in the negative invariant case. We present them here in the most general case of setvalued processes, which were introduced by Roxin [21] under the name general control systems, see also $[8,10,11]$. There is a large literature for autonomous set-valued dynamical systems, which are often called general dynamical systems. Such systems, both autonomous and nonautonomous, are often generated by differential inclusions in the continuous time case (e.g. cf. $[22,15])$ and by set-valued difference equations in the discrete time case (e.g. cf. $[14,16])$. 
Definition 3 A set-valued process on $X$ is given by a mapping $\mathbb{T}_{2}^{\geq} \times X \ni\left(t, t_{0}, x\right) \mapsto$ $\Phi\left(t, t_{0}, x_{0}\right) \in \mathcal{K}(X)$ such that

1) $\Phi\left(t_{0}, t_{0}, x_{0}\right)=\left\{x_{0}\right\}$ for all $x_{0} \in X$ and all $t_{0} \in \mathbb{T}$;

2) $\Phi\left(t_{2}, t_{0}, x_{0}\right)=\Phi\left(t_{2}, t_{1}, \Phi\left(t_{1}, t_{0}, x_{0}\right)\right)$ for all $t_{0} \leq t_{1} \leq t_{2}$ in $\mathbb{T}$ and all $x_{0} \in X$;

3) $\left(t, t_{0}, x_{0}\right) \rightarrow \Phi\left(t, t_{0}, x_{0}\right)$ is upper semi-continuous in $\left(t, t_{0}, x_{0}\right) \in \mathbb{T}_{2}^{\geq} \times X$ with respect to the Hausdorff semi-distance $\operatorname{dist}_{X}$, i.e.

$$
\operatorname{dist}_{X}\left(\Phi\left(s, s_{0}, y_{0}\right), \Phi\left(t, t_{0}, x_{0}\right)\right) \rightarrow 0 \quad \text { as } \quad\left(s, s_{0}, y_{0}\right) \rightarrow\left(t, t_{0}, x_{0}\right) \text { in } \mathbb{T}_{2}^{\geq} \times X ;
$$

4) $\quad t \rightarrow \Phi\left(t, t_{0}, x_{0}\right)$ is continuous in $t \in \mathbb{T}$ (with $t \geq t_{0}$ ) with respect to the Hausdorff metric uniformly in $\left(t_{0}, x_{0}\right)$ in compact subsets of $\mathbb{T} \times X$, i.e.

$$
\sup _{\left(t_{0}, x_{0}\right) \in K} H_{X}\left(\Phi\left(s, t_{0}, x_{0}\right), \Phi\left(t, t_{0}, x_{0}\right)\right) \rightarrow 0 \quad \text { as } s \rightarrow t \text { in } \mathbb{T}
$$

for each $K \in \mathcal{K}(\mathbb{T} \times X)$.

In the autonomous case we simply delete the variable $t_{0}$ and consider $t \in \mathbb{T}^{+}$.

When investigating set-valued processes it is often convenient to consider their single valued trajectories.

Definition 4 A trajectory of a set-valued process is a single valued function $\phi:\left[t_{0}, t_{1}\right] \cap$ $\mathbb{T} \rightarrow X$ for some $\left(t_{1}, t_{0}\right) \in \mathbb{T}_{2}^{\geq}$such that

$$
\phi(t) \in \Phi(t, s, \phi(s)) \quad \text { for all } \quad t_{0} \leq s \leq t \leq t_{1} \quad \text { in } \quad \mathbb{T} .
$$

A trajectory is called an entire trajectory if it is a trajectory on the whole time set $\mathbb{T}$.

In the discrete time case, trajectories are simply parts of sequences. Note that in the continuous time case trajectories are not assumed to be continuous but this follows from the next theorem, which is a generalization of a theorem by Barbashin, see [2,8, 21] for proofs.

Theorem 3 (Barbashin's Theorem) Let $\Phi$ be a set-valued process on a complete metric space $\left(X, d_{X}\right)$. Then

1) there exists a trajectory from $x_{0}$ to $x_{1} \in \Phi\left(t_{1}, t_{0}, x_{0}\right)$ for each $\left(t_{1}, t_{0}\right) \in \mathbb{T}_{2}^{\geq}$and $x_{0}$ $\in X$;

2) trajectories of continuous time set-valued processes are continuous functions, i.e. when $\mathbb{T}=\mathbb{R}$;

3) the set $\mathcal{T}\left(t_{1}, t_{0}, K_{0}\right)$ of all trajectories joining $x_{0}$ to an arbitrary $x_{1} \in \Phi\left(t_{1}, t_{0}, x_{0}\right)$ with $x_{0} \in K_{0}$ is compact in $C\left(\left[t_{0}, t_{1}\right] ; X\right)$ for all $\left(t_{1}, t_{0}\right) \in \mathbb{T}_{2}^{+}$and any nonempty compact subset $K_{0}$ of $X$.

There are two useful types of invariance concepts for set-valued processes, depending on whether the full sets are involved or just certain trajectories. These are called, respectively, strong and weak invariance (e.g. cf. $[24,12,1,2])$.

Definition 5 Given a set-valued process $\Phi$ on $X$, a family $\mathcal{A}=\{A(t): t \in \mathbb{T}\}$ of nonempty sets of $X$ is called $\Phi$-strongly invariant if $\Phi\left(t, t_{0}, A\left(t_{0}\right)\right)=A(t)$ for all $\left(t, t_{0}\right)$ $\in \mathbb{T}_{2}^{\geq} ; \Phi$-strongly positively invariant if $\Phi\left(t, t_{0}, A\left(t_{0}\right)\right) \subset A(t)$ for all $\left(t, t_{0}\right) \in \mathbb{T}_{2}^{\geq}$; and $\Phi$-strongly negatively invariant if $A(t) \subset \Phi\left(t, t_{0}, A\left(t_{0}\right)\right)$ for all $\left(t, t_{0}\right) \in \mathbb{T}_{2}^{\geq}$.

It is called $\Phi$-weakly positively invariant if for every $t_{0} \in \mathbb{T}$ and $a_{0} \in A\left(t_{0}\right)$ there exists a trajectory $\phi:\left[t_{0}, \infty\right) \cap \mathbb{T} \rightarrow X$ with $\phi\left(t_{0}\right)=a_{0}$ such that

$$
\phi(t) \in A(t) \quad \text { for all } t \geq t_{0} \quad \text { in } \quad \mathbb{T} \text {. }
$$


An analogue of the above results for single-valued systems holds for a strongly positively invariant family in the set-valued case, giving a strongly invariant subfamily.

Theorem 4 Let $\Phi$ be a set-valued process on a complete metric space $\left(X, d_{X}\right)$ for the time set $\mathbb{T}$ and let $\mathcal{A}=\{A(t): t \in \mathbb{T}\}$ be a family of nonempty compact subsets of $X$, which is $\Phi$-strongly positively invariant.

Then there exists a family of nonempty compact subsets $\mathcal{A}_{\infty}=\left\{A_{\infty}(t): t \in \mathbb{T}\right\}$ contained in $\mathcal{A}$ in the sense that $A_{\infty}(t) \subset A(t)$ for each $t \in \mathbb{T}$, which is $\Phi$-strongly invariant. The component sets $A_{\infty}(t)$ are given by

$$
A_{\infty}(t)=\bigcap_{t_{0} \leq t} \Phi\left(t, t_{0}, A\left(t_{0}\right)\right), \quad t \in \mathbb{T} .
$$

The proof is similar to that in [13] for the single-valued case. The main difference is that the convergence of $a_{k} \in \Phi\left(t, t_{0}, b_{k}\right)$ to an $\bar{a} \in \Phi\left(t, t_{0}, \bar{b}\right)$ as $b_{k} \rightarrow \bar{b}$ involves the upper semi-continuous convergence in the third variable of $\Phi$, but this suffices to give the desired result. In fact, the proof is the same as that for the existence of a strong nonautonomous pullback attractor once one is inside the strongly positively invariant absorbing set, see $[2,14]$.

\section{Negatively invariant families of compact subsets}

For a strongly negatively invariant family, the subfamily that is obtained in the result below does not need to be strongly invariant, but it is weakly positively invariant.

Theorem 5 Let $\Phi$ be a set-valued process on a complete metric space $\left(X, d_{X}\right)$ for the time set $\mathbb{T}$ and let $\mathcal{A}=\{A(t): t \in \mathbb{T}\}$ be a family of nonempty compact subsets of $X$, which is $\Phi$-strongly negatively invariant.

Then there exists a family of nonempty compact subsets $\mathcal{A}_{\infty}=\left\{A_{\infty}(t): t \in \mathbb{T}\right\}$ which is the maximal $\Phi$-weakly positively invariant family contained in $\mathcal{A}$ in the sense that $A_{\infty}(t) \subset A(t)$ for each $t \in \mathbb{T}$. It is also $\Phi$-strongly negatively invariant.

Remark 2 The family $\mathcal{A}_{\infty}$ here is $\Phi$-strongly negatively invariant like the original family $\mathcal{A}$, but it is also $\Phi$-weakly positively invariant, which the original family does not need to be. Both types of invariances are needed to ensure the existence of entire solutions in $\mathcal{A}_{\infty}$ in the sense that for any $\tau \in \mathbb{T}$ and any $a_{\tau} \in A_{\infty}(\tau)$ there exists an entire trajectory $\phi: \mathbb{T} \rightarrow X$ with $\phi(\tau)=a_{\tau}$ and $\phi(t) \in A_{\infty}(t)$ for all $\tau \in \mathbb{T}$. Specifically, the weak positive invariance gives the existence of a trajectory forwards in time and the strong negative invariance allows us to construct on backwards in time. We then concatenate both parts to obtain an entire trajectory.

We will give the proof of Theorem 5 in several steps as in the corresponding proof for the single valued case considered in [13], going through the discrete time and the continuous time autonomous cases and then the discrete time and the continuous time nonautonomous cases. Indeed, only sections 4.3 and 4.4 are necessary; but sections 4.1 and 4.2 are included for completeness, since we consider a simpler particular situation (not the general case), namely where the family of sets consists of the same set, i.e. $A(t) \equiv A$, since this will allow us to simplify the notation and expose in a more clear way the ideas of the proof in these two first cases. 
4.1 Proof of Theorem 5: Discrete time autonomous systems

Let $\Phi: X \rightarrow \mathcal{K}(X)$ be upper semi-continuous and consider (with the natural but not misleading abuse of notation) the discrete time autonomous set-valued system with $\Phi(n, x):=\Phi^{n}(x)$ for $n \in \mathbb{N}$ and $\Phi(0, x)=\{x\}$. In addition, let $A$ be a nonempty compact subset of $X$ such that $A \subset \Phi(A)$.

Define $A_{0}=A$ and let $A_{-1}$ be the maximal subset of $A_{0}$ such that for each $a_{0} \in$ $A_{0}$ there exists an $a_{-1} \in A_{-1}$ with $a_{0} \in \Phi\left(a_{-1}\right)$, and such that $\Phi\left(a_{-1}\right) \cap A_{0} \neq \emptyset$ for all $a_{-1} \in A_{-1}$.

Then $A_{0} \subset \Phi\left(A_{-1}\right)$. Moreover, $A_{-1}$ is compact by the compactness of $A_{0}$ and the upper semi-continuity of $\Phi$. Indeed, to see this consider sequences $a_{k} \in A_{-1}$ and $b_{k} \in$ $\Phi\left(a_{k}\right) \cap A_{0}$. Since $A_{0}$ is compact and $b_{k} \in A_{0}, a_{k} \in A_{-1} \subset A_{0}$, there are convergent subsequences $a_{k_{j}} \rightarrow \bar{a} \in A_{0}$ and $b_{k_{j}} \rightarrow \bar{b} \in A_{0}$. Then by the upper semi-continuity of $\Phi(\cdot), b_{k_{j}} \in \Phi\left(a_{k_{j}}\right) \rightarrow \Phi(\bar{a})$ with respect to the Hausdorff semi-distance $\operatorname{dist}_{X}$, so $\bar{b} \in$ $\Phi(\bar{a}) \cap A_{0}$, i.e. $\bar{a} \in A_{-1}$, which means that $A_{-1}$ is compact.

Repeating this procedure gives a nested sequence of nonempty compact subsets $A_{-n}, n \geq 0$, contained in $A$, such that for each $a_{-n} \in A_{-n}$ there exists an $a_{-n-1} \in$ $A_{-n-1}$ with $a_{-n} \in \Phi\left(a_{-n-1}\right)$, and such that for any $a_{-n-1} \in A_{-n-1}, \Phi\left(a_{-n-1}\right) \cap A_{-n}$ $\neq \emptyset$. Moreover, $A_{-n-1} \subset A_{-n} \subset \Phi\left(A_{-n-1}\right)$.

Hence the set defined by

$$
A_{\infty}=\bigcap_{n \geq 0} A_{-n}
$$

is a nonempty compact subset of $A$.

Moreover, $A_{\infty}$ is $\Phi$-weakly positively invariant since if $\bar{a} \in A_{\infty}$, then $\bar{a} \in A_{-n-1}$. Thus there exist $b_{n} \in \Phi(\bar{a}) \cap A_{-n}$. Now $\Phi(\bar{a})$ is compact, so there is a convergent subsequence $b_{n_{j}} \rightarrow \bar{b} \in \Phi(\bar{a})$. But $b_{n_{j}} \in A_{-n_{j}}$ and

$$
\operatorname{dist}_{X}\left(\bar{b}, A_{\infty}\right) \leq d_{X}\left(\bar{b}, b_{n_{j}}\right)+\operatorname{dist}_{X}\left(A_{-n_{j}}, A_{\infty}\right) \rightarrow 0,
$$

so $\bar{b} \in A_{\infty}$ and hence $\bar{b} \in A_{\infty} \cap \Phi(\bar{a})$, which gives the desired $\Phi$-weak positive invariance. The maximality of this $\Phi$-weakly positively invariant set is clear by construction.

The set $A_{\infty}$ is also $\Phi$-strongly negatively invariant since if $\bar{a} \in A_{\infty}$, then $\bar{a} \in A_{-n}$ for all $n$, so there exist $b_{n} \in A_{-n}$ such that $\bar{a} \in \Phi\left(b_{n}\right)$. Now the $b_{n} \in A_{0}$, which is compact. Hence there exists a convergent subsequence $b_{n_{j}} \rightarrow \bar{b}$ in $A_{0}$. In fact, $\bar{b} \in A_{\infty}$, since

$$
\operatorname{dist}_{X}\left(\bar{b}, A_{\infty}\right) \leq d_{X}\left(\bar{b}, b_{n_{j}}\right)+\operatorname{dist}_{X}\left(A_{-n_{j}}, A_{\infty}\right) \rightarrow 0 \quad \text { as } j \rightarrow \infty .
$$

Moreover, by upper semi-continuity $\bar{a} \in \Phi\left(b_{n_{j}}\right) \rightarrow \Phi(\bar{b})$ with respect to the Hausdorff semi-distance $\operatorname{dist}_{X}$, so $\bar{a} \in \Phi(\bar{b})$, which means that $A_{\infty} \subset \Phi\left(A_{\infty}\right)$.

\subsection{Proof of Theorem 5: Continuous time autonomous systems}

We consider now a set-valued semi-flow $\Phi: \mathbb{R}_{+} \times X \rightarrow \mathcal{K}(X)$ and a nonempty compact set $A$ of $X$ such that $A \subset \Phi(t, A)$ for all $t \geq 0$.

We apply the result just proved to the discrete time system formed by the time1 mapping $\Phi(1, \cdot): X \rightarrow \mathcal{K}(X)$. This gives us a nonempty compact subset $A_{\infty}^{(1)}$ of $A$ which is the maximal $\Phi(1, \cdot)$-weakly positively invariant subset of $A$, which is also 
$\Phi(1, \cdot)$-strongly negatively invariant. In particular, $\Phi\left(1, a_{\infty}^{(1)}\right) \cap A_{\infty}^{(1)} \neq \emptyset$ for all $a_{\infty}^{(1)} \in$ $A_{\infty}^{(1)}$ and $A_{\infty}^{(1)} \subset \Phi\left(1, A_{\infty}^{(1)}\right)$.

This means that there is a trajectory [in principle defined on $\mathbb{N}$, but extended to $\mathbb{R}^{+}$ by Theorem 3] $\phi^{(1)}: \mathbb{R}^{+} \rightarrow X$ with $\phi^{(1)}(0)=a_{\infty}^{(1)}$ with $\phi^{(1)}(1) \in A_{\infty}^{(1)}$ in particular and $\phi^{(1)}(n) \in A_{\infty}^{(1)}$ for all $n \in \mathbb{N}$. Note that trajectories of $\Phi(1, \cdot)$ are defined only for non-negative integer times, but can be filled in for intermediate times using the continuous time system. The problem is that such a trajectory may not be contained in $A_{\infty}^{(1)}$ for all intermediate times $t \in(0,1)$, etc.

Therefore we repeat the procedure for the discrete time system formed by the time$2^{-1}$ mapping $\Phi\left(2^{-1}, \cdot\right): X \rightarrow \mathcal{K}(X)$ and obtain a nonempty compact subset $A_{\infty}^{(2)}$ of $A$ which is the maximal $\Phi\left(2^{-1}, \cdot\right)$-weakly positively invariant subset of $A$, i.e. for every $a_{\infty}^{(2)} \in A_{\infty}^{(2)}$ there exists a trajectory $\phi^{(2)}: \mathbb{R}^{+} \rightarrow X$ with $\phi^{(2)}(0)=a_{\infty}^{(2)}$ with $\phi^{(2)}\left(2^{-1}\right)$ $\in A_{\infty}^{(2)}$ and $\phi^{(2)}\left(n 2^{-1}\right) \in A_{\infty}^{(2)}$ for all $n \in \mathbb{N}$. In particular, $\phi^{(2)}(1)=\phi^{(2)}\left(2 \cdot 2^{-1}\right) \in$ $A_{\infty}^{(2)}$, so $A_{\infty}^{(2)}$ is also a $\Phi(1, \cdot)$-weakly positively invariant subset of $A$. But $A_{\infty}^{(1)}$ is the maximal compact $\Phi(1, \cdot)$-weakly positively invariant subset of $A$, so $A_{\infty}^{(2)} \subset A_{\infty}^{(1)}$.

Repeat this procedure with the the discrete time system formed by the time- $2^{-n}$ mapping $\Phi\left(2^{-n}, \cdot\right): X \rightarrow \mathcal{K}(X)$ and obtain a nonempty compact subset $A_{\infty}^{(n)}$ of $A$ which is the maximal $\Phi\left(2^{-n}, \cdot\right)$-weakly positively invariant subset of $A$, and thus also $\Phi\left(2^{-n+1}, \cdot\right)$-weakly positively invariant. Hence $A_{\infty}^{(n)} \subset A_{\infty}^{(n-1)}$ for $n=1,2, \ldots$ This is a nested family of nonempty compact subsets, so the set defined by

$$
A_{\infty}=\bigcap_{n \geq 1} A_{\infty}^{(n)}
$$

is a nonempty compact subset of $A$. Moreover, by the discrete time case considered above, $A_{\infty}$ is $\Phi\left(2^{-n}, \cdot\right)$-weakly positively invariant for each $n \in \mathbb{Z}^{+}$. To see this take an arbitrary point $\bar{a} \in A_{\infty}$. Then $\bar{a} \in A_{\infty}^{(n)}$ for each $n \in \mathbb{Z}^{+}$and there exists a trajectory $\phi^{(n)}$ with $\phi^{(n)}(0)=\bar{a}$ and $\phi^{(n)}\left(j 2^{-n}\right) \in A_{\infty}^{(n)}$ for each $j=0,1, \ldots, 2^{n}$. By Barbashin's Theorem 3 the set of all trajectories restricted to the time interval $[0,1]$ of the continuous time system $\Phi$ with initial conditions in the compact set $A_{\infty}$ is compact in $C([0,1] ; X)$, so there is a convergent subsequence $\phi^{\left(n_{j}\right)}$ converging uniformly on $[0,1]$ to a trajectory $\bar{\phi}$. Clearly, $\bar{\phi}(0)=\bar{a}$ and $\bar{\phi}\left(t_{d}\right) \in A_{\infty}$ for all dyadic $t_{d} \in[0,1]$. It follows then by the continuity of $\bar{\phi}$ that $\bar{\phi}(t) \in A_{\infty}$ for all $t \in[0,1]$. This argument can be repeated in the intervals $[1,2],[2,3]$, etc, and then we concatenate the solutions. Thus $A_{\infty}$ is $\Phi$-weakly positively invariant. Again, the maximality of this $\Phi$-weakly positively invariant set is clear by construction.

Finally, we show that $A_{\infty}$ is also $\Phi$-strongly negatively invariant. Indeed, a similar argument to that for the discrete time case applied to the set-valued mapping $\Phi(t, \cdot)$ for a fixed dyadic $t>0$. For a non-dyadic value $t$, proceed by contradiction. Suppose that there exists $\bar{a} \in A_{\infty}$ such that $\bar{a} \notin \Phi\left(t, A_{\infty}\right)$. Take a sequence of dyadic numbers $t_{n}>t$ with $t_{n} \rightarrow t$; since $\bar{a} \in \Phi\left(t_{n}, A_{\infty}\right)$, by Barbashin's Theorem it is possible to take a sequence of trajectories $\phi^{n} \in C\left(\left[0, t_{n}\right] ; X\right)$ with $\phi^{n}\left(t_{n}\right)=\bar{a}$; and since $A_{\infty}$ is compact, we may assume that $\phi^{n}(0)=: b_{n} \rightarrow b$. Then we have $\bar{a} \in \Phi\left(t_{n}, b_{n}\right)$ and by upper semi-continuity, it holds that $\bar{a} \in \Phi(t, b)$. 
4.3 Proof of Theorem 5: Discrete time processes

Fix $n \in \mathbb{Z}$, define $A_{0}^{(j)} \equiv A(j)$ for all $j \in \mathbb{Z}$ and let $A_{-1}^{(n)}$ be the maximal subset of $A(n-1)$ such that for each $a_{0}^{(n)} \in A_{0}^{(n)}$ there exists an $a_{-1}^{(n)} \in A_{-1}^{(n)}$ for which $a_{0}^{(n)} \in \Phi\left(n, n-1, a_{-1}^{(n)}\right)$ and such that for any $a_{-1}^{(n)} \in A_{-1}^{(n)}, \Phi\left(n, n-1, a_{-1}^{(n)}\right) \cap A_{0}^{(n)}$ $\neq \emptyset$. Since $A(n-1)$ is compact and $\Phi(n, n-1, \cdot)$ is upper semi-continuous, the set $A_{-1}^{(n)}$ is compact. Indeed, let be given $\left\{a_{m}^{(n)}\right\}_{m} \subset A_{-1}^{(n)} \subset A(n-1)$. So, there exists a subsequence $a_{m^{\prime}}^{(n)} \rightarrow \bar{a}$. From the definition of $A_{-1}^{(n)}$, there exist $\left\{b_{m}^{(n)}\right\}_{m} \subset A_{0}^{(n)}$ such that $b_{m}^{(n)} \in \Phi\left(n, n-1, a_{m}^{(n)}\right) \cap A_{0}^{(n)}$. Since $A_{0}^{(n)}=A(n)$ is compact, we may assume that $b_{m^{\prime \prime}}^{(n)} \rightarrow \bar{b}$ when $m^{\prime \prime} \rightarrow+\infty$. So, by upper semi continuity, we have that $\bar{b} \in \Phi(n, n-1, \bar{a})$, and therefore $A_{-1}^{(n)}$ is compact.

Note that $A_{-1}^{(n)} \subset A_{0}^{(n-1)}$ and $A_{0}^{(n)} \subset \Phi\left(n, n-1, A_{-1}^{(n)}\right)$.

Repeating this procedure gives a sequence of nonempty compact subsets $A_{-j}^{(n)}$, $j \geq 0$, where $A_{-j-1}^{(n)}$ is the maximal subset in $A(n-j-1)$ such that for each $a_{-j}^{(n)} \in$ $A_{-j}^{(n)}$ there exists an $a_{-j-1}^{(n)} \in A_{-j-1}^{(n)}$ with $a_{-j}^{(n)} \in \Phi\left(n-j, n-j-1, a_{-j-1}^{(n)}\right)$ and such that for any $a_{-j-1}^{(n)} \in A_{-j}^{(n)}, \Phi\left(n-j, n-j-1, a_{-j-1}^{(n)}\right) \cap A_{-j}^{(n)} \neq \emptyset$. Therefore

$$
\Phi\left(n, n-j-1, a_{-j-1}^{(n)}\right) \cap A_{0}^{(n)} \neq \emptyset,
$$

for each $a_{-j-1}^{(n)} \in A_{-j-1}^{(n)}$ for $j=0,1,2, \ldots$ Moreover, $A_{-j}^{(n)} \subset \Phi\left(n-j, n-j-1, A_{-j-1}^{(n)}\right)$.

Similarly, define nonempty compact subsets sets $A_{-j}^{(n+k)}$ for $j, k \in \mathbb{Z}^{+}$. We note that $A_{-k-1}^{(n+k+1)} \subset A_{-k}^{(n+k)}$ for each $k \in \mathbb{Z}^{+}$and fixed $n \in \mathbb{Z}$, i.e. a nested family of nonempty compact subsets of $A(n)$. To see this consider the case $k=1$, recall that $A_{-1}^{(n+1)}$ is the maximal subset of $A(n)$ with $\Phi\left(n+1, n, A_{-1}^{(n+1)}\right) \supset A(n+1)$ and $\Phi\left(n+1, n, a_{-1}^{(n+1)}\right) \cap$ $A(n) \neq \emptyset$ for any $a_{-1}^{(n+1)} \in A_{-1}^{(n+1)}$. Since we also have that $A_{-1}^{(n+2)} \subset \Phi\left(n+1, n, A_{-2}^{(n+2)}\right)$ with $A_{-1}^{(n+2)} \subset A(n+1)$ and $\Phi\left(n+1, n, a_{-2}^{(n+2)}\right) \cap A_{-1}^{(n+2)} \neq \emptyset$ for any $a_{-2}^{(n+2)} \in A_{-2}^{(n+2)}$, from the construction of $A_{-1}^{(n+1)}$ we deduce that $A_{-2}^{(n+2)} \subset A_{-1}^{(n+1)}$.

Hence the set defined by

$$
A_{\infty}(n)=\bigcap_{k \geq 0} A_{-k}^{(n+k)}
$$

is a nonempty compact subset of $A(n)$ for each $n \in \mathbb{Z}$.

Moreover, the family of nonempty compact subsets $\mathcal{A}_{\infty}=\left\{A_{\infty}(n): n \in \mathbb{Z}\right\}$, where $A_{\infty}(n) \subset A(n)$ for each $n \in \mathbb{Z}$, is $\Phi$-weakly positively invariant, since if $\bar{a} \in$ $A_{\infty}(n)$, then $\bar{a} \in A_{-k}^{(n+k)}$, which in particular means that $\Phi(n+1, n, \bar{a}) \cap A_{-k+1}^{(n+k)} \neq \emptyset$. Thus there exist $b_{k} \in \Phi(n+1, n, \bar{a}) \cap A_{-k+1}^{(n+k)}$. Now $\Phi(n+1, n, \bar{a})$ is compact, so there is convergent subsequence $b_{k_{j}} \rightarrow \bar{b} \in \Phi(n+1, n, \bar{a})$. But $b_{k_{j}} \in A_{-k_{j}+1}^{\left(n+k_{j}\right)}=A_{-\left(k_{j}-1\right)}^{\left((n+1)+\left(k_{j}-1\right)\right)}$ $=A_{-l_{j}}^{\left((n+1)+l_{j}\right)}$ with $l_{j}=k_{j}-1$ and

$$
\operatorname{dist}_{X}\left(\bar{b}, A_{\infty}(n+1)\right) \leq d_{X}\left(\bar{b}, b_{k_{j}}\right)+\operatorname{dist}_{X}\left(A_{-l_{j}}^{\left((n+1)+l_{j}\right)}, A_{\infty}(n+1)\right) \rightarrow 0,
$$


so $\bar{b} \in A_{\infty}(n+1)$ and we also had $\bar{b} \in \Phi(n+1, n, \bar{a})$, which gives the desired weak positive invariance. Its maximality, among all $\Phi$-weakly invariant families contained in $\mathcal{A}$, is clear by construction.

Finally, we prove that the family of nonempty compact subsets $\mathcal{A}_{\infty}=\left\{A_{\infty}(n)\right.$ : $n \in \mathbb{Z}\}$ is also $\Phi$-strongly negatively invariant. If $\bar{a} \in A_{\infty}(n)$, then $\bar{a} \in A_{-l}^{(n+l)}$ for all $l$ $\geq 0$. But

$$
A_{-l}^{(n+l)}=A_{-l}^{((n-1)+(l+1))}=A_{-k+1}^{((n-1)+k)}
$$

for $k=l+1 \geq 1$. Thus there exist $b_{k} \in A_{-k}^{((n-1)+k)}$ such that $\bar{a} \in \Phi\left(n, n-1, b_{k}\right)$. Now the $b_{k} \in A(n-1)$, which is compact, so there exists a convergent subsequence $b_{k_{j}} \rightarrow$ $\bar{b}$ in $A(n-1)$. In fact, $\bar{b} \in A_{\infty}(n-1)$, since

$$
\operatorname{dist}_{X}\left(\bar{b}, A_{\infty}(n-1)\right) \leq \operatorname{dist}_{X}\left(\bar{b}, b_{k_{j}}\right)+\operatorname{dist}_{X}\left(A_{-k_{j}}^{\left(n-1+k_{j}\right)}, A_{\infty}(n-1)\right) \rightarrow 0
$$

as $j \rightarrow \infty$. Finally, $\bar{a} \in \Phi(n, n-1, \bar{b})$, since $\bar{a} \in \Phi\left(n, n-1, b_{k}\right) \rightarrow \Phi(n, n-1, \bar{b})$ with respect to the Hausdorff semi-distance $\operatorname{dist}_{X}$ by upper semi-continuity, which implies that $A_{\infty}(n) \subset \Phi\left(n, n-1, A_{\infty}(n-1)\right)$.

\subsection{Proof of Theorem 5: Continuous time processes}

First consider the process restricted the dyadic numbers in $\mathbb{R}$. Let $\mathbb{T}_{0}=\mathbb{Z}$ and $\mathbb{D}_{n}=$ $\left\{d_{j}^{(n)}:=j 2^{-n}: j=0, \ldots, 2^{n}-1\right\}$, then define

$$
\mathbb{T}_{n}:=\mathbb{Z}+\mathbb{D}_{n}=\left\{k+d_{j}^{(n)}: k \in \mathbb{Z}, d_{j}^{(n)} \in \mathbb{D}_{n}\right\}, \quad n=1,2, \ldots
$$

Then we apply the result above to the discrete time set-valued process formed by the restriction $\left.\Phi\right|_{\mathbb{T}_{0}}$ of the set-valued mapping $\Phi$ to the time set $\mathbb{T}_{0}$. This gives us a family $\mathcal{A}_{\infty}^{(0)}=\left\{A_{\infty}^{(0)}(t): t \in \mathbb{T}_{0}\right\}$ of nonempty compact subsets, with $A_{\infty}^{(0)}(t) \subset A(t)$ for each $t \in \mathbb{T}_{0}$, which is the maximal $\left.\Phi\right|_{\mathbb{T}_{0}}$-weakly positively invariant family of subsets of $\left\{A(t): t \in \mathbb{T}_{0}\right\}$, and which is also $\left.\Phi\right|_{\mathbb{T}_{0}}$-strongly negatively invariant, i.e. such that for each $a_{n+1}^{(0)} \in A_{\infty}^{(0)}(n+1)$ there exists an $a_{n}^{(0)} \in A_{\infty}^{(0)}(n)$ for which $a_{n+1}^{(0)} \in \Phi\left(n+1, n, a_{n}^{(0)}\right)$ as well as with $A_{\infty}^{(0)}(n+1) \subset \Phi\left(n+1, n, A_{\infty}^{(0)}(n)\right)$ for any $n \in \mathbb{Z}$.

We will henceforth restrict attention to the case $n=0$ and the time interval $[0,1]$ in the discussion that follows. From above there is a trajectory $\phi^{(0)}:[0,1] \rightarrow X$ of the set-valued process $\Phi$ with $\phi^{(0)}(0)=a_{0}^{(0)} \in A_{\infty}^{(0)}(0)$ and $\phi^{(0)}(1) \in A_{\infty}^{(0)}(1)$. The problem is that such $\phi^{(0)}(t)$ may not be contained in $A(t)$ for all intermediate times $t \in(0,1)$.

Therefore we repeat the procedure for the discrete time system formed by the restriction $\left.\Phi\right|_{\mathbb{T}_{1}}$ of the set-valued mapping $\Phi$ to the time set $\mathbb{T}_{1}$ and obtain a family $\mathcal{A}_{\infty}^{(1)}=\left\{A_{\infty}^{(1)}(t): t \in \mathbb{T}_{1}\right\}$ of nonempty compact subsets, with $A_{\infty}^{(1)}(t) \subset A(t)$, which is the maximal $\left.\Phi\right|_{\mathbb{T}_{1}}$-weakly positively invariant family of subsets of $\{A(t)\}$ as well as being $\left.\Phi\right|_{\mathbb{T}_{1}}$-strongly negatively invariant. In particular, for each $a_{1}^{(1)} \in A_{\infty}^{(1)}(1)$ there exists a trajectory $\phi^{(1)}:[0,1] \rightarrow X$ of $\Phi$ with $\phi^{(1)}(1)=a_{1}^{(1)}$ and $\phi^{(1)}\left(2^{-1}\right) \in A_{\infty}^{(1)}\left(2^{-1}\right)$. In addition, with $A_{\infty}^{(1)}\left(t_{j+1}\right) \subset \Phi\left(t_{j+1}, t_{j}, A_{\infty}^{(1)}\left(t_{j}\right)\right)$ for $t_{j+1}-t_{j}=2^{-1}$. 
By this and the semi-group property,

$$
\begin{aligned}
A_{\infty}^{(1)}(1) & \subset \Phi\left(1,2^{-1}, A_{\infty}^{(1)}\left(2^{-1}\right)\right) \\
& \subset \Phi\left(1,2^{-1}, \Phi\left(2^{-1}, 0, A_{\infty}^{(1)}(0)\right)\right)=\Phi\left(1,0, A_{\infty}^{(1)}(0)\right) .
\end{aligned}
$$

Indeed, this holds on all intervals $[n, n+1]$, so $\mathcal{A}_{\infty}^{(1)}$ is also a $\left.\Phi\right|_{\mathbb{T}_{0}}$-weakly positively invariant and $\left.\Phi\right|_{\mathbb{T}_{0}}$-strongly negatively invariant family of compact sets. But $\mathcal{A}_{\infty}^{(0)}$ is the maximal $\left.\Phi\right|_{\mathbb{T}_{0}}$-weakly positively invariant family of compact subsets of $\{A(t)\}$, so $A_{\infty}^{(1)}(t) \subset A_{\infty}^{(0)}(t)$ for all $t \in \mathbb{T}_{0} \cap \mathbb{T}_{1}=\mathbb{T}_{0}$.

We repeat this procedure with the discrete time system formed by the restriction $\left.\Phi\right|_{\mathbb{T}_{n}}$ of the set-valued-mapping $\Phi$ to the time set $\mathbb{T}_{n}$ and obtain a family $\mathcal{A}_{\infty}^{(n)}=$ $\left\{A_{\infty}^{(n)}(t): t \in \mathbb{T}_{n}\right\}$ of nonempty compact sets, with $A_{\infty}^{(n)}(t) \subset A(t)$, and which is the maximal $\left.\Phi\right|_{\mathbb{T}_{n}}$-weakly positively invariant and $\left.\Phi\right|_{\mathbb{T}_{n}}$-strongly negatively invariant family of subsets of $\{A(t)\}$, and thus also $\left.\Phi\right|_{\mathbb{T}_{n-1}}$-weakly positively invariant and $\left.\Phi\right|_{\mathbb{T}_{n-1}}$ strongly negatively invariant. Hence $A_{\infty}^{(n)}(t) \subset A_{\infty}^{(n-1)}(t)$ for all $t \in \mathbb{T}_{n-1} \cap \mathbb{T}_{n}=\mathbb{T}_{n-1}$, for $n=1,2, \ldots$

Thus for each $t_{l} \in \mathbb{T}_{l}$ for an arbitrary $l \in \mathbb{N}$, the subsets $A_{\infty}^{(n)}\left(t_{l}\right), n \geq l$, are nonempty, compact and nested. Hence the set defined by

$$
A_{\infty}\left(t_{l}\right)=\bigcap_{n \geq l} A_{\infty}^{(n)}\left(t_{l}\right)
$$

is a nonempty compact subset of $A\left(t_{l}\right)$. In this way we obtain a family $\mathcal{A}_{\infty}^{(\text {dyadic })}=$ $\left\{A_{\infty}\left(t_{d}\right): t_{d} \in \cup_{l \geq 0} \mathbb{T}_{l}\right\}$ of nonempty compact sets.

By the discrete time case above, the family $\mathcal{A}_{\infty}^{(\text {dyadic })}$ is also $\left.\Phi\right|_{\mathbb{T}_{n}}$-strongly negatively invariant for each $n=0,1, \ldots$, i.e. with

$$
A_{\infty}\left(t_{j+1}^{(n)}\right) \subset \Phi\left(t_{j+1}^{(n)}, t_{j}^{(n)}, A_{\infty}\left(t_{j}^{(n)}\right)\right)
$$

for every $t_{j+1}^{(n)}, t_{j}^{(n)} \in \mathbb{T}_{n}$ with $t_{j+1}^{(n)}-t_{j}^{(n)}=2^{-n}$. From this and the semi-group property it follows that

$$
A_{\infty}\left(t_{1}\right) \subset \Phi\left(t_{1}, t_{0}, A_{\infty}\left(t_{0}\right)\right)
$$

for all dyadic numbers $t_{0} \leq t_{1}$ in $[0,1]$. Finally, for non-dyadic $t$, we will proceed by density, but this needs to be done in a congruent way.

So we define $A_{\infty}(t)$ to be the cluster points of the sets $A_{\infty}\left(t_{d}\right)$ for dyadic $t_{d} \rightarrow t$ with $t_{d}<t$. We check now that this is well defined. Consider sequences $\left\{t_{d}\right\}$ of dyadic numbers with $t_{d} \uparrow t$, and $\left\{a_{d}\right\}$ with $a_{d} \in A_{\infty}\left(t_{d}\right)$. By (1), $a_{d} \in \Phi\left(t_{d}, t_{1}, A_{\infty}\left(t_{1}\right)\right)$. On other hand, by continuity in the first variable of the process $\Phi$ (property 4 in Definition 3),

$$
H\left(\Phi\left(t_{d}, t_{1}, A_{\infty}\left(t_{1}\right)\right), \Phi\left(t, t_{1}, A_{\infty}\left(t_{1}\right)\right)\right) \rightarrow 0 \quad \text { as } d \rightarrow+\infty .
$$

From this, and the compactness of $\Phi\left(t, t_{1}, A_{\infty}\left(t_{1}\right)\right)$, by the upper semi continuity of $\Phi$, there exists a convergent subsequence $\left\{a_{d^{\prime}}\right\}$ to some element $a \in A_{\infty}(t)$, which is therefore well defined.

Moreover, by a similar argument, one may check that $A_{\infty}(t)$ is closed, and contained in $\Phi\left(t, t_{0}, A_{\infty}\left(t_{0}\right)\right)$, which is compact. So, $A_{\infty}(t)$ is also compact. 
Now we prove that

$$
H_{X}\left(A_{\infty}\left(t_{d}\right), A_{\infty}(t)\right) \rightarrow 0 \quad \text { as } \quad t_{d} \rightarrow t \quad \text { with } \quad t_{d}<t .
$$

The proof follows by contradiction. There are two cases.

1) Suppose that there are an $\varepsilon_{0}>0$ and a sequence of dyadic numbers $t_{d} \rightarrow t$ with $t_{d}$ $<t$ and such that

$$
\varepsilon_{0} \leq \operatorname{dist}_{X}\left(A_{\infty}\left(t_{d}\right), A_{\infty}(t)\right) .
$$

Then there exist $a_{d} \in A_{\infty}\left(t_{d}\right)$ such that

$$
\varepsilon_{0} \leq \operatorname{dist}\left(a_{d}, A_{\infty}(t)\right)=\operatorname{dist}_{X}\left(A_{\infty}\left(t_{d}\right), A_{\infty}(t)\right) .
$$

From the above argument to check that $A_{\infty}(t)$ was closed, we obtained that there exists a convergent subsequence $a_{d^{\prime}} \rightarrow \bar{a}$ in $A_{\infty}(t)$, which is a contradiction.

2) Suppose that there are an $\varepsilon_{0}>0$ and a sequence of dyadic numbers $t_{d} \rightarrow t$ with $t_{d}$ $<t$ and such that

$$
\varepsilon_{0} \leq \operatorname{dist}_{X}\left(A_{\infty}(t), A_{\infty}\left(t_{d}\right)\right) .
$$

Then there exist $a_{d} \in A_{\infty}(t)$ such that

$$
\varepsilon_{0} \leq \operatorname{dist}\left(a_{d}, A_{\infty}\left(t_{d}\right)\right)=\operatorname{dist}_{X}\left(A_{\infty}(t), A_{\infty}\left(t_{d}\right)\right) .
$$

Since $A_{\infty}(t)$ is compact there is a convergent subsequence $a_{d^{\prime}} \rightarrow \bar{a}$ in $A_{\infty}(t)$. By the definition of $A_{\infty}(t)$, there are convergent subsequences $\bar{t}_{d^{\prime}} \uparrow t$ and $\bar{b}_{d^{\prime}} \rightarrow \bar{a}$ with $\bar{b}_{d^{\prime}} \in$ $A_{\infty}\left(\bar{t}_{d^{\prime}}\right)$. We may assume that the sequences $\left\{\bar{t}_{d^{\prime}}\right\}$ and $\left\{t_{d^{\prime}}\right\}$ satisfy that $\bar{t}_{d^{\prime}}<t_{d^{\prime}}<$ $\bar{t}_{(d+1)^{\prime}}$. Since it holds that

$$
\bar{b}_{(d+1)^{\prime}} \in A_{\infty}\left(t_{(d+1)^{\prime}}\right) \subset \Phi\left(\bar{t}_{(d+1)^{\prime}}, t_{d^{\prime}}, A_{\infty}\left(t_{d^{\prime}}\right)\right),
$$

there exist $b_{d^{\prime}} \in A_{\infty}\left(t_{d^{\prime}}\right)$ for all $d^{\prime}$ such that $\bar{b}_{(d+1)^{\prime}} \in \Phi\left(\bar{t}_{(d+1)^{\prime}}, t_{d^{\prime}}, b_{d^{\prime}}\right)$. We may also assume that $b_{d^{\prime}} \rightarrow \bar{c}$. Now, by upper semi-continuity we have that $\bar{b}_{(d+1)^{\prime}} \rightarrow \bar{a} \in$ $\Phi(t, t, \bar{c})=\{\bar{c}\}$. But

$$
\varepsilon_{0} \leq \operatorname{dist}\left(a_{d^{\prime}}, A_{\infty}\left(t_{d^{\prime}}\right)\right) \leq d_{X}\left(a_{d^{\prime}}, \bar{a}\right)+d_{X}\left(\bar{a}, b_{d^{\prime}}\right),
$$

which is a contradiction.

Finally, define $\mathcal{A}_{\infty}=\left\{A_{\infty}(t), t \in \mathbb{R}\right\}$

Now, we check that $\mathcal{A}_{\infty}$ is $\Phi$-weakly positively invariant. We split our analysis in two cases.

1) Firstly consider a dyadic time (without loss of generality we assume $t=0$ ). By the discrete time case above, the family $\mathcal{A}_{\infty}^{\text {(dyadic) }}$ is $\left.\Phi\right|_{\mathbb{T}_{n}}$-weakly positively invariant for each $n=0,1, \ldots$ Indeed, to see this take an arbitrary point $\bar{a} \in A_{\infty}(0)$. Then $\bar{a} \in A_{\infty}^{(n)}(0)$ for each $n \in \mathbb{Z}^{+}$and there exist trajectories $\phi^{(n)}$ with $\phi^{(n)}(0)=\bar{a}$ and $\phi^{(n)}\left(j 2^{-n}\right) \in A_{\infty}^{(n)}\left(j 2^{-n}\right)$ for each $j=0,1, \ldots, 2^{n}$. By the Barbashin's Theorem 3 the set $\mathcal{T}\left(1,0, A_{\infty}(0)\right)$ of all trajectories restricted to the time interval $[0,1]$ of the continuous time system $\Phi$ with initial conditions in the compact set $A_{\infty}(0)$ is compact in $C([0,1] ; X)$, so there is a convergent subsequence $\phi^{\left(n_{j}\right)}$ converging uniformly on $[0,1]$ to a trajectory $\bar{\phi}$. Clearly, $\bar{\phi}(0)=\bar{a}$ and $\bar{\phi}\left(t_{d}\right) \in A_{\infty}\left(t_{d}\right)$ for all dyadic $t_{d} \in[0,1]$. 
Then, from (2) and the continuity of $\bar{\phi}$, it follows that $\bar{\phi}(t) \in A_{\infty}(t)$ for all $t \in[0,1]$. This argument can be repeated in any time interval of the form $[n, n+1]$ and then proceeding by concatenation we obtain the desired result.

2) Now, assume that the time $t$ is non-dyadic, and consider $a \in A_{\infty}(t)$. Let also fix some (dyadic) value $T>t$. From the definition of $A_{\infty}(t), a=\lim a_{d}$ with $a_{d} \in A_{\infty}\left(t_{d}\right)$, where $\left\{t_{d}\right\}_{d}$ is a sequence of dyadic numbers with $t_{d} \uparrow t$. By the case 1$)$, there exist trajectories $\phi^{(d)}$ defined in $\left[t_{d}, T\right]$, and we may consider their restrictions $\left.\phi^{(d)}\right|_{[t, T]}$. Again by Barbashin's Theorem 3 with $K_{0}=\Phi\left(t, t_{1}, A_{\infty}\left(t_{1}\right)\right)$, a subsequence of these functions converge uniformly in $[t, T]$ to a trajectory $\phi$, which satisfies $\phi(t)=a$. As before, this argument can be repeated in any time interval of the form $[T, T+1]$ and then proceeding by concatenation we obtain the desired result.

Its maximality, among all $\Phi$-weakly invariant families contained in $\mathcal{A}$, is clear by construction.

Now we prove that the family $\mathcal{A}_{\infty}$ is $\Phi$-strongly negatively invariant (observe that this was already proved when both $s$ and $t$ are dyadic).

We firstly prove that

$$
A_{\infty}(t) \subset \Phi\left(t, s, A_{\infty}(s)\right) \quad \text { for any } s \text { dyadic and } t \text { arbitrary. }
$$

Indeed, consider a sequence of dyadic numbers $t_{d} \rightarrow t$, and observe that

$$
\begin{aligned}
& \operatorname{dist}_{X}\left(A_{\infty}(t), \Phi\left(t, s, A_{\infty}(s)\right)\right) \\
\leq & \operatorname{dist}_{X}\left(A_{\infty}(t), A_{\infty}\left(t_{d}\right)\right)+\operatorname{dist}_{X}\left(\Phi\left(t_{d}, s, A_{\infty}(s)\right), \Phi\left(t, s, A_{\infty}(s)\right)\right),
\end{aligned}
$$

and by (2) and continuity of $\Phi$ in its first variable, the right hand side goes to zero.

Secondly, we prove the $\Phi$-strongly negatively invariance for the remaining case, i.e. for $s$ non-dyadic. Consider a dyadic sequence $s_{d} \rightarrow s$ with $s_{d}<s$. Then we have that

$$
\begin{aligned}
& \operatorname{dist}_{X}\left(A_{\infty}(t), \Phi\left(t, s, A_{\infty}(s)\right)\right) \\
\leq & \operatorname{dist}_{X}\left(A_{\infty}(t), \Phi\left(t, s_{d}, A_{\infty}\left(s_{d}\right)\right)\right)+\operatorname{dist}_{X}\left(\Phi\left(t, s_{d}, A_{\infty}\left(s_{d}\right)\right), \Phi\left(t, s, A_{\infty}(s)\right)\right),
\end{aligned}
$$

where the first addend in the right hand side is identically zero by (3), and so the second addend converges to zero by the upper semi-continuity of $\Phi$ in its second and third variables and thanks to (2).

To conclude the proof, it only remains to prove that $A_{\infty}(t) \subset A(t)$ for $t$ non-dyadic (for dyadic, the inclusion holds). Observe that for any sequence of dyadic numbers $\left\{t_{d}\right\}_{d}$ with $t_{d} \uparrow d$, from the construction of $A_{\infty}(t)$, it follows that

$$
\begin{aligned}
A_{\infty}(t) & \subset \cap_{t_{d} \uparrow t} \Phi\left(t, t_{d}, A_{\infty}\left(t_{d}\right)\right) \\
& \left.\subset \cap_{t_{d} \uparrow t} \Phi\left(t, t_{d}, A_{(} t_{d}\right)\right) .
\end{aligned}
$$

Since $\{A(t)\}_{t}$ is $\Phi$-strongly negatively invariant, one has that $A(t) \subset \cap_{t_{d} \uparrow t} \Phi\left(t, t_{d}, A\left(t_{d}\right)\right)$. However, we need the opposite inclusion: we will prove that $\cap_{t_{d} \uparrow t} \Phi\left(t, t_{d}, A\left(t_{d}\right)\right) \subset A(t)$. Suppose by a contradiction argument that this is not so. Then, there exists some element $\left.z \in \cap_{t_{d} \uparrow t} \Phi\left(t, t_{d}, A_{(} t_{d}\right)\right) \backslash A(t)$. As $A(t)$ is compact, the distance between $z$ and $A(t)$ is strictly positive, say $\varepsilon=\operatorname{dist}(z, A(t))>0$. Using the weak positive invariance of $\mathcal{A}_{\infty}$, we have that there exists a continuous trajectory $\phi$ with $\phi(t)=z$. Then, for 
an arbitrary and very close dyadic value of time $s>t$, it holds that $d_{X}(\phi(s), z)<\varepsilon / 2$, and therefore

$$
\operatorname{dist}(\phi(s), A(t)) \geq \varepsilon / 2 .
$$

But we know that for any dyadic $s$

$$
\phi(s) \in A_{\infty}(s) \subset A(s),
$$

and by the $\Phi$-strongly negatively invariance of $\{A(t)\}$,

$$
A(s) \subset \Phi(s, t, A(t)) \text {. }
$$

From property 4 of the process $\Phi$, we have that

$$
H(\Phi(s, t, A(t)), A(t)) \rightarrow 0 \quad \text { as } s \rightarrow t .
$$

This contradicts (4)-(6).

The proof of Theorem 5 is completed.

\section{A bifurcation in a set-valued nonautonomous system}

There are at present few results about bifurcations in set-valued dynamical systems, either autonomous or nonautonomous, see [25] and also [17]. The results above allow us to make a preliminary investigation to show that what could be considered that a bifurcation has occurred. This will be illustrated in terms of modifications of a simple example of a pitch fork bifurcation for an scalar ordinary differential equation.

The zero steady state solution of the autonomous semi-dynamical system generated by the differential equation

$$
\frac{d x}{d t}=\nu x-x^{3}
$$

undergoes a supercritical bifurcation at $\nu=0$ to produce two locally asymptotically stable steady state solutions $\pm \sqrt{\nu}$ for $\nu>0$, with the zero steady state solution now unstable.

Let $G: \mathbb{R}^{2} \rightarrow \mathcal{K}([1,2])$ be continuous in the Hausdorff metric and consider the nonautonomous differential inclusion

$$
\frac{d x}{d t} \in \nu x-x^{3}+\varepsilon x G(t, x),
$$

where $\varepsilon>0$ is very small and fixed, which generates a set-valued process $\Phi_{\nu}$ (parameterized by $\nu$ ) with $\Phi_{\nu}\left(t, t_{0}, 0\right)=\{0\}$ for all $\left(t, t_{0}\right) \in \mathbb{R}_{2}^{\geq}$. It also follows that

$$
2(\nu+\varepsilon) x^{2}-2 x^{4} \leq \frac{d}{d t} x^{2} \leq 2(\nu+2 \varepsilon) x^{2}-2 x^{4}
$$

uniformly in $t \in \mathbb{R}$, so the intervals

$$
A_{\varepsilon}^{-}=[-\sqrt{\nu+2 \varepsilon},-\sqrt{\nu+\varepsilon}], \quad A_{\varepsilon}^{+}=[\sqrt{\nu+\varepsilon}, \sqrt{\nu+2 \varepsilon}]
$$

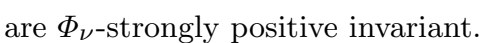

Hence each set $A_{\varepsilon}^{ \pm}$contains invariant families $\mathcal{A}_{\infty}^{ \pm}=\left\{A_{\infty}^{ \pm}(t), t \in \mathbb{R}\right\}$, which one can show that are local pullback (nonautonomous) attractors for the set-valued process $[2,14]$. The zero solution loses stability for some $\nu \in(-\varepsilon, 0)$. The families $\mathcal{A}_{\infty}^{ \pm}$are 
thus candidates for the counterparts of the bifurcating steady state solutions in the autonomous case and provide an indication that some kind of bifurcation has occurred in this nonautonomous set-valued framework.

A second modification may consist in the differential inclusion

$$
\frac{d x}{d t} \in \nu x-x^{3}+\varepsilon G(t, x)
$$

with $G$ as above and $\varepsilon>0$ small enough (namely with $\varepsilon<(2 / 3)^{3 / 2}$ ), which has no zero solution. However, it is not difficult to check that it does have an absorbing and $\Phi_{\nu}$-strongly positively invariant interval $[0, \varepsilon]$, provided that $\nu<\varepsilon^{2}-2$. And therefore, an entire solution exists in that interval.

On the other hand, for $\nu$ big enough, namely, $\nu>\varepsilon^{2}+2$, the interval $[-\varepsilon, \varepsilon]$ is $\Phi_{\nu}$-strongly negatively invariant, and there are two intervals, on either side of this one, which are $\Phi_{\nu}$-strongly positively invariant. Therefore, there exist at least three different entire solutions in this case; and some kind of bifurcation occurs when modifying the parameter $\nu$.

Acknowledgements Partially supported by the Ministerio de Ciencia e Innovación (Spain) grant MTM2008-00088 and Junta de Andalucía grant P07-FQM-02468.

The authors also thank an anonymous referee whose careful reading of an earlier version of the paper has led to improvements in the presentation of the final version.

\section{References}

1. Caraballo T., Kloeden P. E., Marín-Rubio P.: Weak pullback attractors of setvalued processes. J. Math. Anal. Appl. 288, 692-707 (2003).

2. Caraballo T., Kloeden P. E., Marín-Rubio P.: Global and pullback attractors of setvalued skew product flows. Ann. Mat. Pura Appl. (4) 185, S23-S45 (2006).

3. Colombo G., Fěckan M., Garay B.: Multivalued perturbations of a saddle dynamics. Diff. Eq. Dyn. Syst. To appear.

4. Dafermos C. M.: An invariance principle for compact processes. J. Differential Equations 9, 239-252 (1971).

5. Garay B. M., Kloeden P. E.: Discretization near compact invariant sets. Random \& Comput. Dynamics 5, 93-123 (1997).

6. Grüne L.: Asymptotic Behavior of Dynamical and Control Systems under Perturbation and Discretization. Lecture Notes in Mathematics, vol. 1783, Springer-Verlag, Berlin (2002).

7. Hale J.: Asymptotic Behavior of Dissipative Dynamical Systems. Amer. Math. Soc., Providence, RI (1988).

8. Kloeden P. E.: General control systems without backwards extension. In: Differential Games and Control Theory, (Editors: E. Roxin, P. Liu and R. Sternberg), Lecture Notes in Pure Appl. Math., 10, Marcel-Dekker, New York; 49-58 (1974).

9. Kloeden P. E.: Asymptotic invariance and limit sets of general control systems. J. Differential Equations 19, 91-105 (1975).

10. Kloeden P. E.: Eventual stability in general control systems. J. Differential Equations 19, 106-124 (1975)

11. Kloeden P. E.: General control systems. In: Mathematical control theory (Hrsg: W.A. Coppel), Lecture Notes in Mathematics, vol. 680, Springer-Verlag, Berlin, 119-138 (1978).

12. Kloeden P. E., Marín-Rubio P.: Weak pullback attractors of nonautonomous difference inclusions. J. Difference Equ. Appl. 9, 489-502 (2003).

13. Kloeden P. E., Marín-Rubio P.: Negatively invariant sets and entire solutions. Submitted.

14. Kloeden P. E., Schmalfuß B.: Asymptotic behaviour of nonautonomous difference inclusions. Systems \& Control Lett. 33, 275-280 (1998). 
15. Kloeden P. E., Valero J.: Attractors of weakly asymptotically compact set-valued dynamical systems. Set-Valued Anal. 13, 381-404 (2005).

16. Kloeden P. E., Valero J.: Attractors of setvalued partial differential equations under discretization. IMA J. Numer. Anal. To appear.

17. Lamb, J. S. W., Rasmussen, M., and Rodrigues, C. S.: Bifurcations of random maps with bounded noise. Preprint.

18. Mallet-Paret J.: Negatively invariant sets of compact maps and an extension of a theorem of Cartwright. J. Differential Equations 22, 331-348 (1976).

19. Melnik, V. S., Valero, J.: On attractors of multivalued semi-flows and differential inclusions. Set-Valued Anal. 6, 83-111 (1998).

20. Melnik, V. S., Valero, J.: On global attractors of multivalued semiprocesses and nonautonomous evolution inclusions. Set-Valued Anal. 8, 375-403 (2000).

21. Roxin E. O.: Stability in general control systems. J. Differential Equations 1, 115-150 (1965).

22. Roxin E. O.: On generalized dynamical systems defined by contingent equations. J. Differential Equations 1, 188-205 (1965).

23. Sibirsky K. S.: Introduction to Topological Dynamics. Noordhoff International Publishing, Leiden (1975)

24. Szegö G. P., Treccani G.: Semigruppi di Trasformazioni Multivoche. Lecture Notes in Mathematics, vol. 101, Springer-Verlag, Berlin-New York (1969).

25. Tarafdar E. U., Thompson H. B.: Bifurcation for the solutions of equations involving set valued mappings. Internat. J. Math. Math. Sci. 8, 37-48 (1985). 